\title{
Etnobiología en los Andes
}

Rocío AlARCÓN*

\section{RESUMEN}

Partiendo de la revisión de los conceptos básicos sobre etnobiología, se realiza un breve recorrido por la historia de la adaptación cultural y el manejo de los diferentes ambientes en los Andes. Los rápidos procesos de transculturación y la dinámica de la economía de mercado, son los factores utilizados para explicar los cambios ambientales y la pérdida de los conocimientos tradicionales acerca de plantas, animales y sobre el ambiente en general.

Se hace hincapié en la necesidad de tornar a la etnobiología como una importante herramienta científica con múltiples relaciones disciplinarias y diversas utilidades, aunque con una misión primordial: aportar al diseño y aplicación de verdaderas políticas de Desarrollo Sustentable.

Se propone tomar a la etnobiología como matriz de análisis de los problemas del conocimiento tradicional y el ambiente. Sobre esta base es necesario unir voluntades para implementar legislaciones que reconozcan propiedad a los pueblos indígenas y comunidades locales sobre sus conocimientos tradicionales. fax 5932 451338, 339. E-mail: ecoinv@ hoy.net

\begin{abstract}
After reviewing the basic concepts on etnobiology, the author briefly describes the history of cultural adaptation and the use of the different habitats in the Andes. According to the author, the process of cultural adaptation and the dynamics of market economy generate environmental changes and traditional knowledge on plants, animals, and the environment is lost.
\end{abstract}

It is stressed that etnobiology should turn into an important cientific tool that interacts with other disciplines and has different applications. However, its primary mission is to contribute to the design and application of politics centered on sustainable development.

Etnobiology is proposed as the starting point of analysis for traditional knowledge and environmental issues. Therefore, it is necessary to gather efforts to develop laws that recognize the indigenous people's property over their traditional knowledge.

\section{Introducción}

Varios son los puntos de vista vertidos alrededor de esta palabra y varias las disciplinas que deben interactuar para complementar el concepto de etnobiología. Etnobiología comprende el estudio de cómo las diferentes sociedades interactúan con el ambiente natural. Intenta desentrañar cómo las diferentes culturas perciben, clasifican y evalúan los recursos biológicos, y los diferentes caminos seguidos por los diferentes grupos para satisfacer sus propias necesidades. 
Etnobiología es el proceso a través del cual se construye y socializa el conocimiento de los grupos humanos con relación al paisaje natural y sus diversos elementos: plantas, animales, ambientes, etc.

La historia de los Andes está marcada por la presencia de variados grupos humanos que supieron aprovechar los diversos pisos ecológicos presentes en la basta geografía andina. Un evento histórico que ejemplifica esta realidad hasta ahora latente es la expansión de la cultura inca, la cual, bajo una estrategia de sometimiento, logró formar un imperio que integró los conocimientos que poseían las sociedades dominadas acerca de los recursos naturales. De esta manera se llegó a realizar un manejo integral de los recursos naturales creándose el manejo adecuado y diferenciado según el piso ecológico, manejo ahora más conocido como "modelo de microverticalidad andina". La agricultura y el conocimiento de los diversos ambientes fueron las armas que permitieron la formación de un imperio. El intercambio de conocimientos etnobiológicos entre los diferentes grupos y la utilización de éstos fueron sus principales armas de lucha.

Actualmente los grupos indígenas y mestizos, herederos de esa cultura y de otras anteriores, enfrentan un proceso rápido de transculturación, con la consecuente pérdida de conocimientos tradicionales, en especial en lo que hace referencia a los recursos naturales, su utilización y propiedades.

Actualmente el uso integral de los recursos naturales es una quimera, se ha focalizado en productos específicos como el maíz, el ganado o el frijol. La actual economía de mercado ha traído como consecuencia cambios en el conocimiento y el comportamiento de los grupos humanos con relación a la forma de uso y conocimiento de los recursos naturales, y cambios en el patrón de uso del suelo.

\section{Hipótesis}

La etnobiología permite demostrar cómo las diferentes culturas o comunidades podrían integrarse en el proceso de solución de problemas de la conservación, brindando sugerencias fruto de su propia experiencia a través del tiempo, e involu- crándose en el trabajo activo como actores principales en el proceso de implementación de acciones.

\section{Metodología}

Las metas de la conservación en el planeta deben ser compartidas y discutidas por los diferentes grupos humanos en una misma mesa de concertación y la etnobiología es un tema que brinda este espacio.

Escuchar y compartir la experiencia de la gente que habita en el paisaje natural que maneja, manipula, utiliza y explota los recursos naturales.

La etnobiología debe ser vista en el sentido de compartir experiencias, respetar el conocimiento de los diferentes grupos, e integrar en los procesos de trabajo a todos los grupos de interés. A partir de estos consensos se tendrá aun la esperanza de mantener el conocimiento ancestral al servicio de las nuevas generaciones.

\section{Objetivos}

Analizar el rol de la etnobiología con relación a las otras disciplinas.

Incorporar el rol de la etnobiología en el diseño de las políticas de desarrollo y sustentabilidad.

Proponer políticas de propiedad intelectual sobre los recursos genéticos y sobre los conocimientos de los grupos humanos.

\section{El rol de la etnobiología con otras disciplinas}

La etnobiología es una rama que permite abrir un espacio para relacionar dos áreas: el conocimiento ancestral y el conocimiento actual. Permite recopilar toda aquella información ancestral y actual con el fin de levantar una línea de base acerca de una determinada área natural y su relación con las personas que la habitan.

Brinda información a otras disciplinas, pero también abre un espacio para que las otras disciplinas puedan obtener información desde la perspectiva 
de los pobladores que viven en el área en cuestión.

La etnobiología está profundamente relacionada con varias disciplinas, aunque debe su origen principalmente a la biología y a la antropología; sin embargo, la etnobiología tiene múltiples entradas desde ciencias tan diversas como el derecho, la sociología, la geografía o la medicina.

La pregunta es: ¿quién no necesita de la etnobiología para realizar actividades como recabar información sobre territorios ancestrales, vida silvestre, usos y conocimiento de hábitats, usos de suelo, medicina, etc.?

Es menester que al comprender y aceptar el valor de esta disciplina, se la considere como una materia más dentro de las universidades, organizaciones privadas como Ong's y en el ámbito estatal también. Es necesario elaborar métodos adecuados de capacitación en esta disciplina con el fin de valorarla en los procesos de solución de diferentes problemas relacionados con la conservación y el desarrollo.

Varios son los problemas que enfrentamos si no se utiliza la etnobiología como una herramienta. La etnobiología podría ayudar a solucionar algunos problemas tales como:

* El abandono de la tierra y el conocimiento tradicional por parte de los indígenas y campesinos insertos en la economía de mercado y el consumo.

* La migración masiva de campesinos e indígenas a las grandes urbes.

* La pérdida de hábitats y por lo tanto pérdida de especies con diversos usos provocados por un desarrollo sin conservación ni sustentabilidad.

* Falta de interés de las universidades para incluir en su pensum una propuesta etnobiológica a favor de recopilar información de los países con grandes poblaciones indígenas y campesinas.

* Un sistema educativo que no incluye la naturaleza y el conocimiento local diferenciado para los niños de diferentes grupos étnicos: esto se traduce en la pérdida de los propios valores culturales y del ambiente.

\section{Soluciones}

* Orientar los fondos para la investigación a estudios etnobiológicos que se enmarquen en programas de conservación y desarrollo.

* A través de la etnobiología colaborar para lograr injerencia sobre la legislación: reconocimiento de propiedad ancestral y de territorios comunales.

* Integrar en los programas de educación ambiental la información etnobiológica que identifica a los grupos humanos interesados en la realidad de las diferentes zonas.

¿Cómo podemos hablar de sustentabilidad si no involucramos, en los procesos al conocimiento local, a las diversas formas de uso de los recursos, a las diferentes manifestaciones de propiedad de la tierra, etc.?

¿Cómo podemos hablar de sustentabilidad si sólo legislamos, o proponemos normativas sobre la base de la experiencia externa a las zonas en problema y por ende externa a los grupos que allí habitan?

Los grupos humanos son el eslabón final entre el paisaje que ahora tenemos en el planeta y las áreas silvestres. La realidad es que no hay en el modelo ecuatoriano y en América Latina áreas protegidas sin pobladores locales. Por lo tanto es menester que al hablar de sustentabilidad ambiental y social se incluya a estos actores como gestores de un proceso de recuperar información ancestral sobre los hábitats, las formas de propiedad de sus territorios y sobre normativas ancestrales.

La finalidad es que todo este conocimiento sea integrado a procesos de discusión y de aceptación a través del intercambio de experiencias tanto internos como externos, lo que permita el surgimiento de políticas nuevas más reales para las zonas en cuestión y sus habitantes. 


\section{El rol de la etnobiología y las políticas de desarrollo y sustentabilidad}

La gente que habita en las áreas naturales conoce que muchas especies tanto de plantas como de animales tienen valores y usos específicos. Por ejemplo, los campesinos del norte ecuatoriano, cerca del páramo del Angel, provincia del Carchi, mantienen cercas alrededor de sus terrenos sembrados con maíz y pasto. Podríamos pensar que las cercas sirven únicamente para linderar los terrenos y protegerlos de la invasión de ganado vacuno o lanar. Eso es verdad aunque en parte; al preguntar a un grupo de mujeres qué usos tenían los árboles que formaban parte de las cercas, su respuesta fue de que servían como combustible para encender la candela (leña), adicionalmente los arbustos servían para iniciar las llamas y los árboles grandes para mantener un fuego más fuerte y poder coser los alimentos. En el mismo sentido, los hombres contestaron que las cercas servían de refugio a pequeños mamíferos que servían de alimento para su familia y que además, en determinadas épocas del año, en las cercas anidaban aves las cuales también eran fuente de proteína animal.

Es interesante anotar que si tomamos en cuenta el valor individual de las diferentes especies encontradas en el paisaje, se abre la posibilidad de obtener un valor total de la biodiversidad.

A través de la etnobiología podemos encontrar mayor cantidad de productos valiosos y por ende brindar más productos de interés para el mercado. De esta manera podemos proteger una mayor cantidad de especies del bosque sobre la base de la diversidad de productos útiles que oferta el mismo y no sólo focalizar el desarrollo a unos cuantos productos comerciales. Ese es el caso de la provincia de Esmeraldas, en la cual se ofertan solamente cinco o seis especies de maderas comerciales que salen de los bosques; esa presión desmedida sobre estas especies conducirá a la extinción de las mismas. En este contexto vale la pena anotar que el punto central del análisis es que no se puede proteger lo que no se conoce.

Está claro que la mayor biodiversidad encontrada en el país (Ecuador) se debe a la abundancia de especies por unidad de área. De las aproximada- mente 30 mil especies de plantas identificadas en el Ecuador, cinco mil son endémicas. Esta asombrosa biodiversidad se manifiesta también en los diversos y numerosos usos humanos sobre la misma, por ejemplo de las 200 especies de bignoniaceaes identificadas en la zona norte andina, el 38\% tiene usos reconocidos por los pobladores locales.

Usando la etnobiología, a través de sus distintas áreas de investigación se determina el uso actual y potencial de las diferentes especies que forman parte del paisaje natural.

Los mercados locales de las ciudades demuestran que los grupos locales indígenas y campesinos de los Andes manejan un conocimiento profundo de la flora, eso sin contar con las numerosas especies de plantas que son utilizadas únicamente a nivel doméstico y que no son motivo de comercio

Es necesario aplicar la etnobiología como una herramienta que permite recabar conocimientos sobre los recursos naturales. Es menester que a través de la etnobiología se incluya a los actores locales en programas de conservación y desarrollo.

La etnobiología es el enlace entre la conservación y el desarrollo, pues no es posible implementar proyectos de desarrollo sin tomar en cuenta el conocimiento de la historia natural y la forma como los grupos locales han sabido utilizar los recursos.

En el Ecuador los lugares de mayor biodiversidad guardan también una alta diversidad étnica; es importante tomar en cuenta que la etnobiología permite recuperar o realizar un análisis de la biodiversidad encontrada y los usos potenciales que le brinda la gente.

La sustentabilidad ambiental y social se dará en el momento en que se valore la diversidad presente y a su vez se canalicen los conocimientos y la aplicación de los mismos en beneficio de los actores locales quienes de una u otra manera han sido los menos beneficiados históricamente.

\section{Propiedad intelectual}

No se protege lo que no sirve o lo que no se conoce como útil. La historia de América Latina es 
dolorosa en el proceso de reconocimiento sobre el conocimiento ancestral.

A pesar de haber brindado al mundo varias utilidades a través de las plantas y animales nuestro continente no ha sido reconocido en esa perspectiva. Se requiere que se desarrollen políticas que permitan reconocer intelectualmente y pagar con insumos económicos en dos vías: el banco genético y el conocimiento ancestral.

Las decisiones que se tomen sobre la propiedad intelectual tienen implicaciones futuras en la seguridad alimentaria, la agricultura, el desarrollo rural y la conservación de los recursos naturales, y por ende en la biodiversidad.

Se marcará un hito en la historia de los pueblos indígenas y en general en los pueblos o países pobres en el momento en que se reconozcan regalías sobre el conocimiento que poseen su gente con relación a la alta biodiversidad.

El $80 \%$ de la población mundial es dependiente de la medicina tradicional y de las plantas para tratar sus dolencias. Más de dos tercios de las especies vegetales del mundo, de las cuales al menos 35 mil tienen valor medicinal, tienen su origen en los países pobres.

¿No es entonces una manera sustentable social, económica y ambiental el compartir beneficios con los grupos que poseen estos conocimientos?

Se protege lo que se valora, por lo tanto la etnobiología como herramienta permitirá que la sustentabilidad económica, social y ambiental sea una realidad. 